\title{
De novo detection of symmetry in cryo-EM density maps
}

\author{
M. Tykač¹, J. Černý1 ${ }^{1}$ G.N. Murshudov² \\ ${ }^{1}$ Institute of Biotechnology of the Czech Academy of Sciences, Prümyslová 595, 25250 Vestec, Czech Republic, \\ ${ }^{2}$ MRC Laboratory of Molecular Biology, Francis Crick Avenue, Cambridge Biomedical Campus, Cambridge CB2 0QH, United \\ Kingdom
}

Michal.Tykac@ibt.cas.cz

As the number of macromolecular structures solved by the electron microscopy method (EM) rapidly increases, the need for improved methods for processing and improving all aspects of the EM structure determination methods also grows. One possible approach to improving the processing of experimental data is by using the symmetry information. To this end, all major cryo-EM software suites provide an option to use the symmetry information to improve the final resolution of the solved structure. This technique is based on averaging density over all asymmetric units, thus reducing noise and increasing signal to noise ratio in the data.

Nonetheless, all currently available EM suites do require the user to supply the symmetry of the structure and sometimes rotating the structure so that the symmetry axes are in a particular orientation relative to the system axes in order to work. In this contribution, we present a novel method for determining density map symmetries de novo as well as a new software tool called ProSHADE implementing this method.

The presented method relies on computing the optimised self-rotation function [1] using the spherical harmonics decomposition coefficients and converting these onto $\mathrm{SO}(3)$ space coefficients as described by [2]. By subsequently computing the inverse Fourier transform in $\mathrm{SO}(3)$ space, the self-rotation function is obtained. Next, the self-rotation function values are mapped onto a set of concentric spheres with radius equal to the angle of rotation in the axis-angle rotation representation, while the position on the sphere represents the rotation axis of the axis-angle rotation representation. This representation allows for fast detection of any axis which has high self-rotation function values along any particular set of angles. This in turn allows for detection of cyclic (C) symmetry groups as they are by definition a set of rotations, along the same axis, which do not change the shape (i.e. have high self-rotation function value). Once all C symmetries are detected, the dihedral (D), tetrahedral (T), octahedral (O) and icosahedral (I) symmetries can be detected by finding for the required $\mathrm{C}$ symmetries combinations forming the larger symmetry groups.

Since the self-rotation function values are proportional to real-space correlation between the original and rotated density map values, they are affected by the shape of the density map; this relationship is such that the more spherical the density map is, the higher the overall real-space correlation will be irrespective of the actual symmetry in the density. Therefore, to reduce the number of false positive results, the method also uses the Fourier Shell Correlation (FSC) to confirm any detected symmetry, increasing the reliability of the method.

The current implementation of this method in ProSHADE is capable of correctly detecting the symmetry type and fold for over $85 \%$ of symmetrised structures deposited in the EMDB database [3] with approximately half of the incorrectly determined symmetries being a subgroup of the originally reported symmetry. The symmetry detection does not require any user input and is therefore readily available for inclusion into cryo-EM structure solving pipelines. ProSHADE is an open source project available under the GPL version 3 license on all major operating systems either as stand-alone executable or as a python language module.

[1] Navaza J. (1994). Acta Cryst, A50, 157-163.

[2] Kostelec P.J., Rockmore D.N. (2008). Journal of Fourier Analysis and Applications, 14, 145-179.

[3] Lawson C.L., Baker M.L., Best C., Bi C., Dougherty M., Feng P., van Ginkel G., Devkota B., Lagerstedt I., Ludtke S.J., Newman R.H., Oldfield, T.J., Rees I., Sahni G., Sala R., Velankar S., Warren J., Westbrook J.D., Henrick K., Kleywegt G.J., Berman H.M., and Chiu W.C. (2011). Nucleic Acids Research, 39, 456-464.

Keywords: Symmetry, cryo-EM 